\title{
Note on Extending Congruential Modal Logics
}

\author{
Lloyd Humberstone
}

\begin{abstract}
It is observed that a consistent congruential modal logic is not guaranteed to have a consistent extension in which the Box operator becomes a truthfunctional connective for one of the four one-place (two-valued) truth functions.
\end{abstract}

\section{Background}

Let us fix the language of propositional (mono)modal logic as having countably many sentence letters $\left(p_{1}, \ldots, p_{n}, \ldots\right)$ and primitive connectives $\rightarrow, \square$, and $\perp$ of arities 2,1 , and 0 , respectively, subject to the usual formation rules; ${ }^{1}$ other connectives, in particular $\top, \neg, \wedge$, and $\leftrightarrow$, are taken as defined in the familiar ways. A modal logic is a set of formulas in this language which contains all truth-functional tautologies and is closed under uniform substitution and modus ponens, and is said to be consistent if it does not contain every formula (equivalently, does not contain $\perp$ ). A modal logic $S$ is monotone, antitone, or congruential, respectively, when $A \rightarrow B \in S$ implies $\square A \rightarrow \square B \in S$ (for all formulas $A, B$ ), or $A \rightarrow B \in S$ implies $\square B \rightarrow \square A \in S$ (all formulas $A, B$ ), or $A \leftrightarrow B \in S$ implies $\square A \leftrightarrow \square B \in S$ (again: all $A, B$ ). (This terminology is taken from Makinson [14] and [15].) Finally, the term truth function refers throughout to two-valued truth functions (similarly with cognate vocabulary, such as truth-functional), though we sometimes include a parenthetical reminder to that effect.

Theorem 1 of Makinson [14] tells us that if $S$ is any consistent congruential modal logic containing $\square \top$ and $\neg \square \perp$, then $S$ can be consistently extended by the addition of all formulas $\square A \leftrightarrow A$. Calling the modal logic containing all such formulas the identity logic, Makinson's formulation is that any consistent congruential $S$ containing $\square \top$ and $\neg \square \perp$ is a sublogic of the identity logic- - which is itself a consistent

Received July 27, 2013; accepted October 4, 2013

First published online September 30, 2015

2010 Mathematics Subject Classification: Primary 03B45

Keywords: modal logic, Post completeness, congruential modal logics, neighborhood semantics

C 2016 by University of Notre Dame $\quad 10.1215 / 00294527-3315588$ 


\title{
Note on Extending Congruential Modal Logics
}

\author{
Lloyd Humberstone
}

\begin{abstract}
It is observed that a consistent congruential modal logic is not guaranteed to have a consistent extension in which the Box operator becomes a truthfunctional connective for one of the four one-place (two-valued) truth functions.
\end{abstract}

\section{Background}

Let us fix the language of propositional (mono)modal logic as having countably many sentence letters $\left(p_{1}, \ldots, p_{n}, \ldots\right)$ and primitive connectives $\rightarrow, \square$, and $\perp$ of arities 2,1 , and 0 , respectively, subject to the usual formation rules; ${ }^{1}$ other connectives, in particular $\top, \neg, \wedge$, and $\leftrightarrow$, are taken as defined in the familiar ways. A modal logic is a set of formulas in this language which contains all truth-functional tautologies and is closed under uniform substitution and modus ponens, and is said to be consistent if it does not contain every formula (equivalently, does not contain $\perp$ ). A modal logic $S$ is monotone, antitone, or congruential, respectively, when $A \rightarrow B \in S$ implies $\square A \rightarrow \square B \in S$ (for all formulas $A, B$ ), or $A \rightarrow B \in S$ implies $\square B \rightarrow \square A \in S$ (all formulas $A, B$ ), or $A \leftrightarrow B \in S$ implies $\square A \leftrightarrow \square B \in S$ (again: all $A, B$ ). (This terminology is taken from Makinson [14] and [15].) Finally, the term truth function refers throughout to two-valued truth functions (similarly with cognate vocabulary, such as truth-functional), though we sometimes include a parenthetical reminder to that effect.

Theorem 1 of Makinson [14] tells us that if $S$ is any consistent congruential modal logic containing $\square \top$ and $\neg \square \perp$, then $S$ can be consistently extended by the addition of all formulas $\square A \leftrightarrow A$. Calling the modal logic containing all such formulas the identity logic, Makinson's formulation is that any consistent congruential $S$ containing $\square \top$ and $\neg \square \perp$ is a sublogic of the identity logic- - which is itself a consistent

Received July 27, 2013; accepted October 4, 2013

First published online September 30, 2015

2010 Mathematics Subject Classification: Primary 03B45

Keywords: modal logic, Post completeness, congruential modal logics, neighborhood semantics

C 2016 by University of Notre Dame $\quad 10.1215 / 00294527-3315588$ 


\title{
Note on Extending Congruential Modal Logics
}

\author{
Lloyd Humberstone
}

\begin{abstract}
It is observed that a consistent congruential modal logic is not guaranteed to have a consistent extension in which the Box operator becomes a truthfunctional connective for one of the four one-place (two-valued) truth functions.
\end{abstract}

\section{Background}

Let us fix the language of propositional (mono)modal logic as having countably many sentence letters $\left(p_{1}, \ldots, p_{n}, \ldots\right)$ and primitive connectives $\rightarrow, \square$, and $\perp$ of arities 2,1 , and 0 , respectively, subject to the usual formation rules; ${ }^{1}$ other connectives, in particular $\top, \neg, \wedge$, and $\leftrightarrow$, are taken as defined in the familiar ways. A modal logic is a set of formulas in this language which contains all truth-functional tautologies and is closed under uniform substitution and modus ponens, and is said to be consistent if it does not contain every formula (equivalently, does not contain $\perp$ ). A modal logic $S$ is monotone, antitone, or congruential, respectively, when $A \rightarrow B \in S$ implies $\square A \rightarrow \square B \in S$ (for all formulas $A, B$ ), or $A \rightarrow B \in S$ implies $\square B \rightarrow \square A \in S$ (all formulas $A, B$ ), or $A \leftrightarrow B \in S$ implies $\square A \leftrightarrow \square B \in S$ (again: all $A, B$ ). (This terminology is taken from Makinson [14] and [15].) Finally, the term truth function refers throughout to two-valued truth functions (similarly with cognate vocabulary, such as truth-functional), though we sometimes include a parenthetical reminder to that effect.

Theorem 1 of Makinson [14] tells us that if $S$ is any consistent congruential modal logic containing $\square \top$ and $\neg \square \perp$, then $S$ can be consistently extended by the addition of all formulas $\square A \leftrightarrow A$. Calling the modal logic containing all such formulas the identity logic, Makinson's formulation is that any consistent congruential $S$ containing $\square \top$ and $\neg \square \perp$ is a sublogic of the identity logic- - which is itself a consistent

Received July 27, 2013; accepted October 4, 2013

First published online September 30, 2015

2010 Mathematics Subject Classification: Primary 03B45

Keywords: modal logic, Post completeness, congruential modal logics, neighborhood semantics

C 2016 by University of Notre Dame $\quad 10.1215 / 00294527-3315588$ 


\title{
Note on Extending Congruential Modal Logics
}

\author{
Lloyd Humberstone
}

\begin{abstract}
It is observed that a consistent congruential modal logic is not guaranteed to have a consistent extension in which the Box operator becomes a truthfunctional connective for one of the four one-place (two-valued) truth functions.
\end{abstract}

\section{Background}

Let us fix the language of propositional (mono)modal logic as having countably many sentence letters $\left(p_{1}, \ldots, p_{n}, \ldots\right)$ and primitive connectives $\rightarrow, \square$, and $\perp$ of arities 2,1 , and 0 , respectively, subject to the usual formation rules; ${ }^{1}$ other connectives, in particular $\top, \neg, \wedge$, and $\leftrightarrow$, are taken as defined in the familiar ways. A modal logic is a set of formulas in this language which contains all truth-functional tautologies and is closed under uniform substitution and modus ponens, and is said to be consistent if it does not contain every formula (equivalently, does not contain $\perp$ ). A modal logic $S$ is monotone, antitone, or congruential, respectively, when $A \rightarrow B \in S$ implies $\square A \rightarrow \square B \in S$ (for all formulas $A, B$ ), or $A \rightarrow B \in S$ implies $\square B \rightarrow \square A \in S$ (all formulas $A, B$ ), or $A \leftrightarrow B \in S$ implies $\square A \leftrightarrow \square B \in S$ (again: all $A, B$ ). (This terminology is taken from Makinson [14] and [15].) Finally, the term truth function refers throughout to two-valued truth functions (similarly with cognate vocabulary, such as truth-functional), though we sometimes include a parenthetical reminder to that effect.

Theorem 1 of Makinson [14] tells us that if $S$ is any consistent congruential modal logic containing $\square \top$ and $\neg \square \perp$, then $S$ can be consistently extended by the addition of all formulas $\square A \leftrightarrow A$. Calling the modal logic containing all such formulas the identity logic, Makinson's formulation is that any consistent congruential $S$ containing $\square \top$ and $\neg \square \perp$ is a sublogic of the identity logic- - which is itself a consistent

Received July 27, 2013; accepted October 4, 2013

First published online September 30, 2015

2010 Mathematics Subject Classification: Primary 03B45

Keywords: modal logic, Post completeness, congruential modal logics, neighborhood semantics

C 2016 by University of Notre Dame $\quad 10.1215 / 00294527-3315588$ 


\title{
Note on Extending Congruential Modal Logics
}

\author{
Lloyd Humberstone
}

\begin{abstract}
It is observed that a consistent congruential modal logic is not guaranteed to have a consistent extension in which the Box operator becomes a truthfunctional connective for one of the four one-place (two-valued) truth functions.
\end{abstract}

\section{Background}

Let us fix the language of propositional (mono)modal logic as having countably many sentence letters $\left(p_{1}, \ldots, p_{n}, \ldots\right)$ and primitive connectives $\rightarrow, \square$, and $\perp$ of arities 2,1 , and 0 , respectively, subject to the usual formation rules; ${ }^{1}$ other connectives, in particular $\top, \neg, \wedge$, and $\leftrightarrow$, are taken as defined in the familiar ways. A modal logic is a set of formulas in this language which contains all truth-functional tautologies and is closed under uniform substitution and modus ponens, and is said to be consistent if it does not contain every formula (equivalently, does not contain $\perp$ ). A modal logic $S$ is monotone, antitone, or congruential, respectively, when $A \rightarrow B \in S$ implies $\square A \rightarrow \square B \in S$ (for all formulas $A, B$ ), or $A \rightarrow B \in S$ implies $\square B \rightarrow \square A \in S$ (all formulas $A, B$ ), or $A \leftrightarrow B \in S$ implies $\square A \leftrightarrow \square B \in S$ (again: all $A, B$ ). (This terminology is taken from Makinson [14] and [15].) Finally, the term truth function refers throughout to two-valued truth functions (similarly with cognate vocabulary, such as truth-functional), though we sometimes include a parenthetical reminder to that effect.

Theorem 1 of Makinson [14] tells us that if $S$ is any consistent congruential modal logic containing $\square \top$ and $\neg \square \perp$, then $S$ can be consistently extended by the addition of all formulas $\square A \leftrightarrow A$. Calling the modal logic containing all such formulas the identity logic, Makinson's formulation is that any consistent congruential $S$ containing $\square \top$ and $\neg \square \perp$ is a sublogic of the identity logic- - which is itself a consistent

Received July 27, 2013; accepted October 4, 2013

First published online September 30, 2015

2010 Mathematics Subject Classification: Primary 03B45

Keywords: modal logic, Post completeness, congruential modal logics, neighborhood semantics

C 2016 by University of Notre Dame $\quad 10.1215 / 00294527-3315588$ 


\title{
Note on Extending Congruential Modal Logics
}

\author{
Lloyd Humberstone
}

\begin{abstract}
It is observed that a consistent congruential modal logic is not guaranteed to have a consistent extension in which the Box operator becomes a truthfunctional connective for one of the four one-place (two-valued) truth functions.
\end{abstract}

\section{Background}

Let us fix the language of propositional (mono)modal logic as having countably many sentence letters $\left(p_{1}, \ldots, p_{n}, \ldots\right)$ and primitive connectives $\rightarrow, \square$, and $\perp$ of arities 2,1 , and 0 , respectively, subject to the usual formation rules; ${ }^{1}$ other connectives, in particular $\top, \neg, \wedge$, and $\leftrightarrow$, are taken as defined in the familiar ways. A modal logic is a set of formulas in this language which contains all truth-functional tautologies and is closed under uniform substitution and modus ponens, and is said to be consistent if it does not contain every formula (equivalently, does not contain $\perp$ ). A modal logic $S$ is monotone, antitone, or congruential, respectively, when $A \rightarrow B \in S$ implies $\square A \rightarrow \square B \in S$ (for all formulas $A, B$ ), or $A \rightarrow B \in S$ implies $\square B \rightarrow \square A \in S$ (all formulas $A, B$ ), or $A \leftrightarrow B \in S$ implies $\square A \leftrightarrow \square B \in S$ (again: all $A, B$ ). (This terminology is taken from Makinson [14] and [15].) Finally, the term truth function refers throughout to two-valued truth functions (similarly with cognate vocabulary, such as truth-functional), though we sometimes include a parenthetical reminder to that effect.

Theorem 1 of Makinson [14] tells us that if $S$ is any consistent congruential modal logic containing $\square \top$ and $\neg \square \perp$, then $S$ can be consistently extended by the addition of all formulas $\square A \leftrightarrow A$. Calling the modal logic containing all such formulas the identity logic, Makinson's formulation is that any consistent congruential $S$ containing $\square \top$ and $\neg \square \perp$ is a sublogic of the identity logic- - which is itself a consistent

Received July 27, 2013; accepted October 4, 2013

First published online September 30, 2015

2010 Mathematics Subject Classification: Primary 03B45

Keywords: modal logic, Post completeness, congruential modal logics, neighborhood semantics

C 2016 by University of Notre Dame $\quad 10.1215 / 00294527-3315588$ 


\title{
Note on Extending Congruential Modal Logics
}

\author{
Lloyd Humberstone
}

\begin{abstract}
It is observed that a consistent congruential modal logic is not guaranteed to have a consistent extension in which the Box operator becomes a truthfunctional connective for one of the four one-place (two-valued) truth functions.
\end{abstract}

\section{Background}

Let us fix the language of propositional (mono)modal logic as having countably many sentence letters $\left(p_{1}, \ldots, p_{n}, \ldots\right)$ and primitive connectives $\rightarrow, \square$, and $\perp$ of arities 2,1 , and 0 , respectively, subject to the usual formation rules; ${ }^{1}$ other connectives, in particular $\top, \neg, \wedge$, and $\leftrightarrow$, are taken as defined in the familiar ways. A modal logic is a set of formulas in this language which contains all truth-functional tautologies and is closed under uniform substitution and modus ponens, and is said to be consistent if it does not contain every formula (equivalently, does not contain $\perp$ ). A modal logic $S$ is monotone, antitone, or congruential, respectively, when $A \rightarrow B \in S$ implies $\square A \rightarrow \square B \in S$ (for all formulas $A, B$ ), or $A \rightarrow B \in S$ implies $\square B \rightarrow \square A \in S$ (all formulas $A, B$ ), or $A \leftrightarrow B \in S$ implies $\square A \leftrightarrow \square B \in S$ (again: all $A, B$ ). (This terminology is taken from Makinson [14] and [15].) Finally, the term truth function refers throughout to two-valued truth functions (similarly with cognate vocabulary, such as truth-functional), though we sometimes include a parenthetical reminder to that effect.

Theorem 1 of Makinson [14] tells us that if $S$ is any consistent congruential modal logic containing $\square \top$ and $\neg \square \perp$, then $S$ can be consistently extended by the addition of all formulas $\square A \leftrightarrow A$. Calling the modal logic containing all such formulas the identity logic, Makinson's formulation is that any consistent congruential $S$ containing $\square \top$ and $\neg \square \perp$ is a sublogic of the identity logic- - which is itself a consistent

Received July 27, 2013; accepted October 4, 2013

First published online September 30, 2015

2010 Mathematics Subject Classification: Primary 03B45

Keywords: modal logic, Post completeness, congruential modal logics, neighborhood semantics

C 2016 by University of Notre Dame $\quad 10.1215 / 00294527-3315588$ 


\title{
Note on Extending Congruential Modal Logics
}

\author{
Lloyd Humberstone
}

\begin{abstract}
It is observed that a consistent congruential modal logic is not guaranteed to have a consistent extension in which the Box operator becomes a truthfunctional connective for one of the four one-place (two-valued) truth functions.
\end{abstract}

\section{Background}

Let us fix the language of propositional (mono)modal logic as having countably many sentence letters $\left(p_{1}, \ldots, p_{n}, \ldots\right)$ and primitive connectives $\rightarrow, \square$, and $\perp$ of arities 2,1 , and 0 , respectively, subject to the usual formation rules; ${ }^{1}$ other connectives, in particular $\top, \neg, \wedge$, and $\leftrightarrow$, are taken as defined in the familiar ways. A modal logic is a set of formulas in this language which contains all truth-functional tautologies and is closed under uniform substitution and modus ponens, and is said to be consistent if it does not contain every formula (equivalently, does not contain $\perp$ ). A modal logic $S$ is monotone, antitone, or congruential, respectively, when $A \rightarrow B \in S$ implies $\square A \rightarrow \square B \in S$ (for all formulas $A, B$ ), or $A \rightarrow B \in S$ implies $\square B \rightarrow \square A \in S$ (all formulas $A, B$ ), or $A \leftrightarrow B \in S$ implies $\square A \leftrightarrow \square B \in S$ (again: all $A, B$ ). (This terminology is taken from Makinson [14] and [15].) Finally, the term truth function refers throughout to two-valued truth functions (similarly with cognate vocabulary, such as truth-functional), though we sometimes include a parenthetical reminder to that effect.

Theorem 1 of Makinson [14] tells us that if $S$ is any consistent congruential modal logic containing $\square \top$ and $\neg \square \perp$, then $S$ can be consistently extended by the addition of all formulas $\square A \leftrightarrow A$. Calling the modal logic containing all such formulas the identity logic, Makinson's formulation is that any consistent congruential $S$ containing $\square \top$ and $\neg \square \perp$ is a sublogic of the identity logic- - which is itself a consistent

Received July 27, 2013; accepted October 4, 2013

First published online September 30, 2015

2010 Mathematics Subject Classification: Primary 03B45

Keywords: modal logic, Post completeness, congruential modal logics, neighborhood semantics

C 2016 by University of Notre Dame $\quad 10.1215 / 00294527-3315588$ 


\title{
Note on Extending Congruential Modal Logics
}

\author{
Lloyd Humberstone
}

\begin{abstract}
It is observed that a consistent congruential modal logic is not guaranteed to have a consistent extension in which the Box operator becomes a truthfunctional connective for one of the four one-place (two-valued) truth functions.
\end{abstract}

\section{Background}

Let us fix the language of propositional (mono)modal logic as having countably many sentence letters $\left(p_{1}, \ldots, p_{n}, \ldots\right)$ and primitive connectives $\rightarrow, \square$, and $\perp$ of arities 2,1 , and 0 , respectively, subject to the usual formation rules; ${ }^{1}$ other connectives, in particular $\top, \neg, \wedge$, and $\leftrightarrow$, are taken as defined in the familiar ways. A modal logic is a set of formulas in this language which contains all truth-functional tautologies and is closed under uniform substitution and modus ponens, and is said to be consistent if it does not contain every formula (equivalently, does not contain $\perp$ ). A modal logic $S$ is monotone, antitone, or congruential, respectively, when $A \rightarrow B \in S$ implies $\square A \rightarrow \square B \in S$ (for all formulas $A, B$ ), or $A \rightarrow B \in S$ implies $\square B \rightarrow \square A \in S$ (all formulas $A, B$ ), or $A \leftrightarrow B \in S$ implies $\square A \leftrightarrow \square B \in S$ (again: all $A, B$ ). (This terminology is taken from Makinson [14] and [15].) Finally, the term truth function refers throughout to two-valued truth functions (similarly with cognate vocabulary, such as truth-functional), though we sometimes include a parenthetical reminder to that effect.

Theorem 1 of Makinson [14] tells us that if $S$ is any consistent congruential modal logic containing $\square \top$ and $\neg \square \perp$, then $S$ can be consistently extended by the addition of all formulas $\square A \leftrightarrow A$. Calling the modal logic containing all such formulas the identity logic, Makinson's formulation is that any consistent congruential $S$ containing $\square \top$ and $\neg \square \perp$ is a sublogic of the identity logic- - which is itself a consistent

Received July 27, 2013; accepted October 4, 2013

First published online September 30, 2015

2010 Mathematics Subject Classification: Primary 03B45

Keywords: modal logic, Post completeness, congruential modal logics, neighborhood semantics

C 2016 by University of Notre Dame $\quad 10.1215 / 00294527-3315588$ 